\title{
Novel Efficient Routes to Heparin Monosaccharides, and Disaccharides Achieved via Regio- and Stereoselective Glycosidation
}

\section{Henry N. Yu, Jun-ichi Furukawa, Tsuyoshi Ikeda and Chi-Huey Wong*}

\author{
Department of Chemistry and Skaggs Institute for Chemical Biology, The Scripps \\ Research Institute, 10550 North Torrey Pines Road, La Jolla, California 92037
}

General. All chemicals were purchased as reagent grade and used without further purification. Dichloromethane $\left(\mathrm{CH}_{2} \mathrm{Cl}_{2}\right)$, toluene, and acetonitrile $\left(\mathrm{CH}_{3} \mathrm{CN}\right)$ were distilled over calcium hydride whereas tetrahydrofuran (THF) and ether $\left(\mathrm{Et}_{2} \mathrm{O}\right)$ were distilled over sodium/benzophenone ketyl. Anhydrous DMF was purchased from a commercial source. Molecular sieves (MS) used for glycosylation were AW-300, which was ground into powdered form before use. Reactions were monitored with analytical thin-layer chromatography (TLC) on silica gel 60 F254 plates and visualized under UV (254 nm) and/or by staining with $5 \%$ sulfuric acid or acidic ceric ammonium molybdate. ${ }^{1} \mathrm{H}$ NMR spectra were recorded on a $400-$ or $500-\mathrm{Hz}$ NMR spectrometer at $20{ }^{\circ} \mathrm{C}$. Chemical shift (in ppm) was determined relative to either tetramethylsilane in deuterated chloroform $(\delta 0$ ppm) or acetone in deuterated water ( $\delta 2.05 \mathrm{ppm})$. Coupling constant(s) in hertz $(\mathrm{Hz})$ were measured from one-dimensional spectra. ${ }^{13} \mathrm{C}$ Attached Proton Test (C-Apt) spectra were obtained with NMR-400 or NMR 500 spectrometer and were calibrated with $\mathrm{CDCl}_{3}$ ( $\delta 77.00 \mathrm{ppm})$. Mass spectra were obtained by the analytical services of this department. 
Methyl 1,2,3,4-tetra- $O$-acetyl-5- $C$-bromo- $\alpha$-D-glucopuranosyluronate (6a) - A suspension of methyl 1,2,3,4-tetra- $O$-accetyl- $\alpha$-D-glucopyranuroate 5a (4.7 g, 12.5 mmol) and $N$-bromosuccinimide $(3.5 \mathrm{~g}, 19.7 \mathrm{mmol})$ in dry $\mathrm{CCl}_{4}(160 \mathrm{~mL})$ was stirred for 45 min under irradiation with two $250 \mathrm{~W}$ tungsten light blubs (reflux). The mixture was cooled to room temperature and filtered by Celite, and the solvent was removed by evaporation. Short column chromatography (5:1 toluene-EtOAc) of residue gave the amorphous compound 6a (4.0 g, $70 \%) ;{ }^{1} \mathrm{H}$ NMR: $\delta 6.54(\mathrm{~d}, 1 \mathrm{H}, J 4.1, \mathrm{H}-1), 5.81$ (dd, 1H, $J$ 9.9, 10.3, H-3), 5.31 (d, 1H, J 9.4, H-4), 5.19 (dd, 1H, J 4.1, 10.3, H-2), 3.83 (s, $\left.3 \mathrm{H}, \mathrm{COOCH}_{3}\right), 2.21,2.11,2.06,2.05$ (s x 4, 12H, 4 x Ac); ${ }^{13} \mathrm{C} \mathrm{NMR}\left(\mathrm{CDCl}_{3}\right): \delta 169.5$, 169.3, 168.8, 168.5, 164.7 (C=O x 5), 89.4 (C-1), 87.1 (C-5), 69.7 (C-4), 67.8 (C-2), 66.9 (C-3), 54.1 ( $\left.\mathrm{OCH}_{3}\right), 21.1,20.6,20.6,20.5$ (Ac x 4); m/z (High Res. MALDI-FTMS) calcd for $\mathrm{C}_{15} \mathrm{H}_{19} \mathrm{BrO}_{11} \mathrm{Na}\left(\mathrm{M}+\mathrm{Na}^{+}\right)$477.0003, found: 477.0003 .

\section{Methyl 1,2,3,4-tetra- $O$-acetyl-5- $C$-bromo- $\beta$-D-glucopuranosyluronate $\quad(6 \mathrm{~b})$} Compound $\mathbf{5 b}$ was converted to $\mathbf{6 b}$ as described above. ${ }^{1} \mathrm{H}$ NMR: $\delta 6.28(\mathrm{~d}, 1 \mathrm{H}, J$ 8.6, H1), 5.54 (t, 1H, J 9.5, H-3), 5.33 (d, 1H, J 9.4, H-4), 5.25 (dd, 1H, J 8.8, 9.7, H-2), 3.83 (s, $\left.3 \mathrm{H}, \mathrm{COOCH}_{3}\right), 2.28,2.21,2.06,2.03$ (s x 4, 12H, 4 x Ac).

Methyl 1,2,3,4-tetra-O-acetyl- $\alpha$-L-idopyranuroate (7a) - A mixture of bromide 6a (3.9 $\mathrm{g}, 8.5 \mathrm{mmol})$ and tributyltin hydride $(4.5 \mathrm{~mL}, 16.7 \mathrm{mmol})$ in dry benzene was stirred at $80{ }^{\circ} \mathrm{C}$ under Ar, AIBN (47 mg, $0.29 \mathrm{mmol}$ ) was added to the solution. The reaction was stirred for $2 \mathrm{~h}$ and allowed to cool to room temperature. The solvent was evaporated and residue was diluted with EtOAc and saturated potassium fluoride was added. The mixture 
was filtered by celite pad. The filtrate was washed with saturated aqueous $\mathrm{NaHCO}_{3}$ and brine. The organic layer was dried $\left(\mathrm{Na}_{2} \mathrm{SO}_{4}\right)$ and concentrated. Column chromatography (2:1 toluene-EtOAc) of residue gave amorphous compound 7a (4.0 g, $70 \%) . \quad{ }^{1} \mathrm{H}$ NMR: $\delta 6.05(\mathrm{~d}, 1 \mathrm{H}, J 1.8, \mathrm{H}-1), 5.28(\mathrm{t}, 1 \mathrm{H}, J 3.3,3.7, \mathrm{H}-3), 5.10(\mathrm{ddd}, 1 \mathrm{H}, J$ 0.7, 2.9, 2.6, H4), 5.19 (ddd, $1 \mathrm{H}, J$ 0.7, 1.8, 2.9, H-2), $4.72(\mathrm{~d}, 1 \mathrm{H}, J 2.2, \mathrm{H}-5), 3.78\left(\mathrm{~s}, 3 \mathrm{H}, \mathrm{COOCH}_{3}\right)$, 2.17, 2.14, 2.11, 2.09 (s x 4, 12H, 4 x Ac) $;{ }^{13} \mathrm{C} \mathrm{NMR}\left(\mathrm{CDCl}_{3}\right): \delta 169.4,169.2,168.6$, 168.1, $166.8(\mathrm{C}=\mathrm{O} \times$ x ), 89.7 (C-1), 73.3 (C-5), $67.2(\mathrm{C}-3), 66.2$ (C-4), 65.2 (C-2), 52.7 (OCH3), 20.7, 20.7, 20.7, 20.5 (Ac x 4);m/z (High Res. MALDI-FTMS) calcd for $\mathrm{C}_{15} \mathrm{H}_{20} \mathrm{O}_{11} \mathrm{Na}\left(\mathrm{M}+\mathrm{Na}^{+}\right)$399.08985, found: 399.0887 .

Methyl 1,2,3,4-tetra- $\boldsymbol{O}$-acetyl- $\beta$-L-idopyranuroate (7b) - Compound $\mathbf{6 b}$ was converted to $7 \mathbf{b}(53 \%)$ as described above. ${ }^{1} \mathrm{H}$ NMR: $\delta 6.29\left(\mathrm{dd}, 1 \mathrm{H}, J_{1,3} 1.3,1.9 \mathrm{H}-1\right), 5.18(\mathrm{t}, 1 \mathrm{H}$, $J$ 3.6, H-4), 5.16 (td, $\left.1 \mathrm{H}, J_{1,3} 1.0,4.0, \mathrm{H}-3\right), 4.88$ (d, $1 \mathrm{H}, J$ 2.5, H-5), 4.86 (ddd, $1 \mathrm{H}, J_{2,4}$ $1.0,2.3,3.4, \mathrm{H}-2), 3.80\left(\mathrm{~s}, 3 \mathrm{H}, \mathrm{COOCH}_{3}\right), 2.13,2.12,2.11,2.09$ (s x 4, 12H, 4 x Ac); m/z (High Res. MALDI-FTMS) calcd for $\mathrm{C}_{15} \mathrm{H}_{20} \mathrm{O}_{11} \mathrm{Na}\left(\mathrm{M}+\mathrm{Na}^{+}\right)$399.0898, found: 399.0897.

Methyl (2,3,4-tetra-O-acetyl- $\alpha$-L-idopyranosyl bromide)uroate (8) - A solution of acetylated iduronic acid $7 \mathbf{a}(1.5 \mathrm{~g}, 4.0 \mathrm{mmol})$ in $30 \% \mathrm{HBr}$ solution in acetic acid $(20 \mathrm{~mL})$ was stirred for $2 \mathrm{~h}$ at $0{ }^{\circ} \mathrm{C}$ to room temperature. The mixture was evaporated with toluene. The residue was diluted with EtOAc and washed with cold saturated aqueous $\mathrm{NaHCO}_{3}$ and brine. The organic layer extraction was dried $\left(\mathrm{Na}_{2} \mathrm{SO}_{4}\right)$ and concentrate to give the bromide 8 (1.6 g, quant). Spectral data were in good agreement with those reported. ${ }^{13 a}$ 
Methyl 3,4-di-O-acetyl-1,2-O-(S)-ethylidene- $\alpha$-L-idopyranuroate (9) - To solution of bromide 8 (1.5 g, $3.8 \mathrm{mmol})$ in $\mathrm{MeCN}$ (40 mL), $\mathrm{NaBH}_{4}(0.9 \mathrm{~g}, 23.8 \mathrm{mmol})$ was added at $0{ }^{\circ} \mathrm{C}$ and stirred for $2 \mathrm{~h}$ at room temperature. The reaction mixture was filtered by Celite pad and concentrated. Chromatography of the residue on silica gel with Hexane:EtOAc (2:1) gave the 1,2-acetal derivative 9 (0.96 g, $70 \%) .{ }^{1} \mathrm{H}$ NMR: $\delta 5.42(\mathrm{t}, 1 \mathrm{H}, J 2.2, \mathrm{H}-3)$, 5.37 (t, 1H, J 2.6, H-1), 5.34 (q, 1H, J 4.75, CH), 5.08 (m, 1H, H-4), 4.45 (d, 1H, J 1.45, H-5), 3.78 (s, 3H, $\left.\mathrm{COOCH}_{3}\right), 3.76$ (bs, 1H, H-2), 2.15, 2.07 (s x 2, $6 \mathrm{H}, 2$ x Ac), 1.54 (d, $\left.1 \mathrm{H}, J 4.75, \mathrm{CH}_{3}\right) .{ }^{13} \mathrm{C} \mathrm{NMR}\left(\mathrm{CDCl}_{3}\right): \delta 169.6,168.4,167.5,(\mathrm{C}=\mathrm{O} \times 3), 104.5,95.6(\mathrm{C}-1)$, 75.0 (C-5), 70.3, 66.5, 66.4, 52.6 (OCH3), 21.7, 20.7, 20.5; m/z (High Res. MALDIFTMS) calcd for $\mathrm{C}_{13} \mathrm{H}_{18} \mathrm{O}_{9} \mathrm{Na}\left(\mathrm{M}+\mathrm{Na}^{+}\right)$341.0843, found: 341.0840 .

Methyl 1,2-O-(S)-ethylidene- $\alpha$-L-idopyranuroate (10) - To solution of acetate 9 (487 mg, $1.5 \mathrm{mmol})$ in $\mathrm{MeOH}(20 \mathrm{~mL}), \mathrm{NaOMe}$ (cat.) was added and stirred for $3 \mathrm{~h}$. The reaction was neutralized by DOWEX 650C, filtered and concentrated. Chromatography of the residue on silica gel with Hexane:EtOAc (2:1) gave derivative 10 (402 mg, 95 \%). ${ }^{1} \mathrm{H}$ NMR: $\delta 5.40(\mathrm{~d}, 1 \mathrm{H}, J$ 2.0, H-1), 5.28 (q, 1H, J 5.0, CH), 4.55 (bs, 1H, H-5), 4.48 (m, 1H, H-3), 3.96-3.99 (m, 1H, J 12.1, H-4), 3.84 (dd, 1H, J 2.0, H-2), 3.77 (s, 3H, $\left.\mathrm{COOCH}_{3}\right), 3.06\left(\mathrm{~d}, 1 \mathrm{H}, J\right.$ 12.1, 4-OH), $2.45(\mathrm{~m}, 1 \mathrm{H}, 3-\mathrm{OH}), 1.56\left(\mathrm{~d}, 1 \mathrm{H}, J 5.0, \mathrm{CH}_{3}\right) ;{ }^{13} \mathrm{C}$ NMR ( $\left.\mathrm{CDCl}_{3}\right): \delta 169.7(\mathrm{C}=\mathrm{O}), 103.9(\mathrm{CH}), 95.5(\mathrm{C}-1), 78.4(\mathrm{C}-2), 71.4(\mathrm{C}-5), 69.6(\mathrm{C}-4)$, $66.7(\mathrm{C}-3), 52.5\left(\mathrm{OCH}_{3}\right), 21.7\left(\mathrm{CH}_{3}\right) ; \mathrm{m} / \mathrm{z}$ (High Res. MALDI-FTMS) calcd for $\mathrm{C}_{9} \mathrm{H}_{14} \mathrm{O}_{7} \mathrm{Na}\left(\mathrm{M}+\mathrm{Na}^{+}\right)$257.0632. found: 257.0630 . 


\section{$p$-methylphenyl $\quad 2$-azido-4,6- $O$-benzylidene-2-deoxy-3-O-p-methoxybenzyl-1-thio- $\beta$ -}

D-glucopyranoside (11) - To a mixture of mannoside $32(640 \mathrm{mg}, 1.29 \mathrm{mmol})$ in pyridine-dichloromethane $(1: 1,12 \mathrm{~mL})$ at $-15{ }^{\circ} \mathrm{C}$ under argon, was added liquid trifluoromethanesulfonic anhydride $(1 \mathrm{~mL})$. The reaction was allowed to warm to room temperature. The reaction was diluted with EtOAc and quenched with a few drops of $\mathrm{H}_{2} \mathrm{O}$. The mixture was then washed with $\mathrm{HCl}(2 \mathrm{~N}), \mathrm{NaHCO}_{3}$, and brine. The organic layer was dried over $\mathrm{Na}_{2} \mathrm{SO}_{4}$ and concentrated in vacuo. The residue was then stirred in anhydrous DMF at $0{ }^{\circ} \mathrm{C}$ under argon. Solid $\mathrm{NaN}_{3}(422 \mathrm{mg}, 6.48 \mathrm{mmol})$ was added. The reaction was allowed to warm to room temperature and proceed overnight. The reaction was diluted with EtOAc, washed with $\mathrm{H}_{2} \mathrm{O}$. The organic layer was dried over $\mathrm{Na}_{2} \mathrm{SO}_{4}$ and concentrated in vacuo. The resulting residue was purified by column chromatography (3\% EtOAc in toluene) to give 11 (537 mg, 80\%). ${ }^{1} \mathrm{H}$ NMR (400 MHz, $\left.\mathrm{CDCl}_{3}\right) \delta 7.49-$ $6.84(\mathrm{~m}, 9 \mathrm{H}, \mathrm{PMB}+\mathrm{Ph}), 5.55(\mathrm{~s}, 1 \mathrm{H}, \mathrm{CHPh}), 4.83(\mathrm{~d}, 1 \mathrm{H}, J$ 10.5, PMB), $4.70(\mathrm{~d}, 1 \mathrm{H}, J$ 10.5, PMB), 4.40 (d, 1H, $J$ 10.3, H-1), 4.17 (dd, 1H, $J 4.8,10.5, \mathrm{H}-6 \mathrm{a}), 3.77$ (overlapped, 4H, H-6b + OMe), 3.63 ('t', 1H, J 8.6, H-3), 3.57 ('t', 1H, J 9.1, H-4), 3.42 (d't', 1H, $J$ 5.1, 9.7, H-5), 3.30 (dd, $1 \mathrm{H}, J 8.9,10.1, \mathrm{H}-2), 2.33$ (s, 3H, Tol). ${ }^{13} \mathrm{C}\left(400 \mathrm{MHz}, \mathrm{CDCl}_{3}\right) \delta$ $159.7,139.4,137.4,134.8,134.3,130.3,130.1,129.9,129.7,129.3,128.5,126.6,126.2$, 114.1, 114.0, 101.4, 86.7, 81.5, 80.8, 75.1, 10.7, 68.7, 64.6, 55.5, 21.4. m/z (High Res. MALDI-FTMS) calcd $\mathrm{C}_{28} \mathrm{H}_{29} \mathrm{~N}_{3} \mathrm{O}_{5} \mathrm{SNa}^{+}: 542.1720$, found: 542.1722 .

Methyl 4-O-acetyl-1,2-O-(S)-ethylidene- $\beta$-L-idopyranosyluronate (16) - Under argon, $\operatorname{Mg}(\mathrm{OMe})_{2}(25 \mu \mathrm{L}, 6-10 \mathrm{wt} . \%)$ was added dropwise to a stirred solution of 9 (30 mg, $94.3 \mu \mathrm{mol})$ in anhydrous $\mathrm{MeOH}$-dichloromethane $(1: 1,1 \mathrm{~mL})$ at $0{ }^{\circ} \mathrm{C}$, and reaction was 
continued for $2.5 \mathrm{~h}$. $\mathrm{MeOH}(10 \mathrm{~mL})$ was added and the mixture was neutralized to $\mathrm{pH} 7$ with $\mathrm{H}^{+}$resin. After filtration, the mixture was concentrated. The residue was purified by chromatography on silica using toluene - EtOAc $(1: 1 \mathrm{v} / \mathrm{v})$ as eluent to give compound 16 (23 mg, 88\%) and 9 (3.6 mg, 12\%). ${ }^{1} \mathrm{H}$ NMR (400 MHz, $\left.\mathrm{CDCl}_{3}\right) \delta 5.41(\mathrm{~d}, J 2.6,1 \mathrm{H}, \mathrm{H}-$ 1), 5.33 (q, $1 \mathrm{H}, J 5.0, \mathrm{CHCH}_{3}$ ), 5.02 (ddd, $\left.1 \mathrm{H}, J 1.3,1.3,2.6, \mathrm{H}-4\right), 4.61(\mathrm{~d}, 1 \mathrm{H}, J 1.5, \mathrm{H}-$ 5), 4.45 ('t', $1 \mathrm{H}, J 2.5 \mathrm{H}-3), 3.83(\mathrm{~m}, 1 \mathrm{H}, \mathrm{H}-2), 3.80\left(\mathrm{~s}, 3 \mathrm{H}, \mathrm{CO}_{2} \mathrm{CH}_{3}\right), 2.12(\mathrm{~s}, 3 \mathrm{H}, \mathrm{OAc})$, $1.10\left(\mathrm{~d}, 3 \mathrm{H}, J\right.$ 5.0, $\left.\mathrm{CHCH}_{3}\right) .{ }^{13} \mathrm{C}\left(400 \mathrm{MHz}, \mathrm{CDCl}_{3}\right) \delta 169.9,167.6,104.4,95.9,77.7$, 70.0, 69.7, 65.6, 52.8, 22.0, 21.1; m/z (High Res. MALDI-FTMS) calcd $\mathrm{C}_{11} \mathrm{H}_{16} \mathrm{O}_{8} \mathrm{Na}^{+}$: 299.0737, found: 299.0735 .

\section{Methyl 4-O-acetyl-3-O-benzoyl-1,2- $O$ - $(S)$-ethylidene- $\beta$-L-idopyranosyluronate (17) -}

Under argon, benzoyl chloride $(200 \mu \mathrm{L})$ was added dropwise to a stirred solution of $\mathbf{1 6}$ (22 $\mathrm{mg}, 80 \mu \mathrm{mol})$ in pyridine $(1 \mathrm{~mL})$ at $0{ }^{\circ} \mathrm{C}$. The reaction was continued at room temperature for $30 \mathrm{~min}$. The mixture was concentrated and the resulting residue was purified by chromatography on silica using toluene - EtOAc $(7: 3 \mathrm{v} / \mathrm{v})$ as eluent to give compound 17 (26 mg, 86\%). ${ }^{1} \mathrm{H}$ NMR (400 MHz, $\left.\mathrm{CDCl}_{3}\right) \delta 8.13-7.48$ (m, 5H, Bz), 5.68 $(\mathrm{d}, J 1.8,1 \mathrm{H}, \mathrm{H}-1), 5.45(\mathrm{~d}, 1 \mathrm{H}, J 2.3 \mathrm{H}-3), 5.38\left(\mathrm{q}, 1 \mathrm{H}, J 4.8, \mathrm{CHCH}_{3}\right), 5.25(\mathrm{dd}, 1 \mathrm{H}, J$ 1.5, 2.5, H-4), 4.52 (s, $1 \mathrm{H}, \mathrm{H}-5), 3.82(\mathrm{~s}, 1 \mathrm{H}, \mathrm{H}-2), 3.78\left(\mathrm{~s}, 3 \mathrm{H}, \mathrm{CO}_{2} \mathrm{CH}_{3}\right), 2.11(\mathrm{~s}, 3 \mathrm{H}$,

OAc), 1.07 (d, 3H, J 5.0, $\left.\mathrm{CHCH}_{3}\right) .{ }^{13} \mathrm{C}\left(400 \mathrm{MHz}, \mathrm{CDCl}_{3}\right) \delta 167.0,167.8,164.3,134.1$, $134.0,130.4,130.1,128.9,128.7,104.9,96.0,75.4,70.8,67.2,66.8,52.9,22.0,20.9$; m/z (High Res. MALDI-FTMS) calcd $\mathrm{C}_{18} \mathrm{H}_{20} \mathrm{O}_{9} \mathrm{Na}^{+}$: 403.0999, found: 403.1004 . 


\section{Methyl 4-O-acetyl-3-O-pivaloyl-1,2-O-(S)-ethylidene- $\beta$-L-idopyranosyluronate (18) -}

Under argon, trimethyl acetyl chloride $(150 \mu \mathrm{L})$ was added dropwise to a stirred solution of $16(22 \mathrm{mg}, 80 \mu \mathrm{mol})$ and DMAP (cat.) in pyridine $(1 \mathrm{~mL})$ at $0{ }^{\circ} \mathrm{C}$. The ice-bath was removed and reaction was continued at room temperature for $12 \mathrm{~h}$. The mixture was concentrated and the resulting residue was purified by chromatography on silica using toluene - EtOAc (5:1 v/v) as eluent to give compound $18(23 \mathrm{mg}, 80 \%) .{ }^{1} \mathrm{H}$ NMR (400 $\left.\mathrm{MHz}, \mathrm{CDCl}_{3}\right) \delta 5.40$ ('t', 1H, J 2.3, H-3), 5.35 (q, 1H, J 4.8, $\left.\mathrm{CHCH}_{3}\right), 5.33(\mathrm{~d}, 1 \mathrm{H}, J 2.8$, H-1), 5.06 (dd, 1H, J 1.3, 2.4, H-4), 4.43 (d, 1H, J 1.3, H-5), 3.78 (s, 3H, $\left.\mathrm{CO}_{2} \mathrm{CH}_{3}\right), 3.71$ (s, 1H, H-2), 2.08 (s, 3H, OAc), 1.55 (d, 3H, J 4.7, $\left.\mathrm{CHCH}_{3}\right), 1.24$ (s, 9H, Piv); ${ }^{13} \mathrm{C}(400$ $\left.\mathrm{MHz}, \mathrm{CDCl}_{3}\right) \delta 176.0,169.8,167.8,104.9,96.0,75.3,70.7,66.7,66.3,52.8,39.0,27.2$ 22.0, 20.8; m/z (High Res. MALDI-FTMS) calcd $\mathrm{C}_{16} \mathrm{H}_{24} \mathrm{O}_{9} \mathrm{Na}^{+}$: 383.1313, found: 383.1313.

Methyl 3,4-di-O-acetyl-1,2-O -ethylidene- $\alpha$-D-glucopuranosyluronate (20) - To а stirring mixture of bromide $19(50 \mathrm{mg}, 126 \mu \mathrm{mol})$, tetrabutylammonium iodide $(30 \mathrm{mg}$, $81 \mu \mathrm{mol})$ and activated $3 \AA$ molecular sieves in anhydrous $\mathrm{CH}_{3} \mathrm{CN}(2 \mathrm{~mL})$, solid $\mathrm{NaBH}_{4}$ (30 mg, $793 \mu \mathrm{mol}$ ) was added. The reaction was heated at $40^{\circ} \mathrm{C}$ for $16 \mathrm{~h}$. The mixture was then diluted with dichloromethane and filtered. After evaporation, the residue was was purified by chromatography on silica using toluene - EtOAc $(7: 3 \mathrm{v} / \mathrm{v})$ as eluent to give compound 20 (4 mg, 10\%). ${ }^{1} \mathrm{H}$ NMR (400 MHz, $\left.\mathrm{CDCl}_{3}\right) \delta 5.64(\mathrm{~d}, 1 \mathrm{H}, J 4.9, \mathrm{H}-1)$, $5.20(\mathrm{dd}, 1 \mathrm{H}, J 2.9,4,7, \mathrm{H}-3), 5.11$ (dd, 1H, J 2.8, 9.0, H-4), 5.07 (q, 1H, J 4.9, $\mathrm{CHCH}_{3}$ ), 4.37 (d, 1H, J 8.8 H-5), 4.02 (dd, 1H, J 3.0, 4.5, H-2), $3.71\left(\mathrm{~s}, 3 \mathrm{H}, \mathrm{CO}_{2} \mathrm{CH}_{3}\right), 2.08(\mathrm{~s}, 3 \mathrm{H}$, 
OAc), 2.06 (s, 3H, OAc), 1.50 (d, 3H, $J 4.9, \mathrm{CHCH}_{3}$ ). (High Res. MALDI-FTMS) calcd $\mathrm{C}_{13} \mathrm{H}_{18} \mathrm{O}_{9} \mathrm{Na}^{+}: 341.0843$, found: 341.0845 .

Methyl 1,2-O-(S)-ethylidene- $\alpha$-D-glucopuranosyluronate (25a) - A solution of $\mathrm{NaOCl}$ (8.1 mL, $\mathrm{d}=1.097)$, saturated $\mathrm{NaHCO}_{3}(5.8 \mathrm{~mL})$ and brine $(9.5 \mathrm{~mL})$ at $0{ }^{\circ} \mathrm{C}$ was added dropwise for $2 \mathrm{~h}$ to a cooled solution of $\mathbf{2 4 a}(500 \mathrm{mg}, 2.42 \mathrm{mmol})$, TEMPO (12.9 mg, $0.83 \mathrm{mmol}$ ), sodium bromide (51 $\mathrm{mg}, 0.50 \mathrm{mmol}$ ), tetrabuytlammonium chloride (64 $\mathrm{mg}$, $0.23 \mathrm{mmol})$, in saturated $\mathrm{NaHCO}_{3}(9.5 \mathrm{~mL})$ and EtOAc $(16 \mathrm{~mL})$. The reaction was stirred overnight at $4{ }^{\circ} \mathrm{C}$. The mixture was washed with EtOAc and extracted with $\mathrm{H}_{2} \mathrm{O}(4 \mathrm{X})$. The aqueous layers was combined and acidified to $\mathrm{pH} 1$ with $\mathrm{HCl}(2 \mathrm{~N})$. The mixture was then concentrated, co-evaporated with toluene and dried in vacuo. The resulting residue was then stirred in anhydrous $\mathrm{MeOH}$ with $\mathrm{H}^{+}$resin for $36 \mathrm{~h}$. The resin was removed by filtration and organic solvent removed. The residue was then diluted with EtOAc washed with $\mathrm{H}_{2} \mathrm{O}$. The organic layer was dried over $\mathrm{Na}_{2} \mathrm{SO}_{4}$ and concentrated in vacuo. The resulting residue was purified by column chromatography using toluene - EtOAc (1:9 $\mathrm{v} / \mathrm{v}$ ) as eluent to give compound 25a (351 $\mathrm{mg}, 62 \%) .{ }^{1} \mathrm{H} \mathrm{NMR}\left(400 \mathrm{MHz}, \mathrm{CDCl}_{3}\right) \delta 5.71$ (d, $J 4.1,1 \mathrm{H}, \mathrm{H}-1), 5.54$ (q, $\left.1 \mathrm{H}, J 5.0, \mathrm{CHCH}_{3}\right), 4.31(\mathrm{~d}, 1 \mathrm{H}, J 7.3 \mathrm{H}-5), 4.12(\mathrm{dd}, 1 \mathrm{H}, J$ 4.1, 5.7, $\mathrm{H}-2), 3.98$ (dd, $1 \mathrm{H}, J 3.2,5.9, \mathrm{H}-3), 3.84$ (m, 4H, $\left.\mathrm{H}-4+\mathrm{CO}_{2} \mathrm{CH}_{3}\right), 1.39$ (d, $3 \mathrm{H}, J$ 4.8, $\left.\mathrm{CHCH}_{3}\right) .{ }^{13} \mathrm{C}\left(400 \mathrm{MHz}, \mathrm{CDCl}_{3}\right) \delta 171.0,102.2,96.5,78.5,76.9,74.2,70.6,69.8$, 52.8, 20.6; m/z (High Res. ESI) calcd $\mathrm{C}_{9} \mathrm{H}_{14} \mathrm{O}_{7} \mathrm{Cl}^{-}: 269$, found: 269 .

Methyl 1,2-O-(R)-ethylidene- $\alpha$-D-glucopuranosyluronate (25b) - Compound 24b was converted to $\mathbf{2 5 b}$ as described above. ${ }^{1} \mathrm{H}$ NMR $\left(400 \mathrm{MHz}, \mathrm{CDCl}_{3}\right) \delta 5.52(\mathrm{~d}, J 3.7,1 \mathrm{H}$, 
$\mathrm{H}-1), 5.18\left(\mathrm{q}, 1 \mathrm{H}, J\right.$ 5.7, $\left.\mathrm{CHCH}_{3}\right), 4.40(\mathrm{~d}, 1 \mathrm{H}, J$ 6.7, H-5), 4.16 (m, 1H, H-3), 4.05 (dd, $1 \mathrm{H}, J 3.7,6.7, \mathrm{H}-2), 4.0(\mathrm{~m}, 1 \mathrm{H}, \mathrm{H}-4), 3.82\left(\mathrm{~s}, 3 \mathrm{H}, \mathrm{CO}_{2} \mathrm{CH}_{3}\right), 1.50\left(\mathrm{~d}, 3 \mathrm{H}, J 4.8, \mathrm{CHCH}_{3}\right)$. ${ }^{13} \mathrm{C}\left(400 \mathrm{MHz}, \mathrm{CDCl}_{3}\right) \delta 171.2,102.4,95.8,73.7,73.4,70.6,70.3,52.8,20.6 ; \mathrm{m} / \mathrm{z}$ (GC/MS) calcd $\mathrm{C}_{9} \mathrm{H}_{14} \mathrm{O}_{7}^{+}: 234$, found: 234.

\section{2-azido-4,6-O-benzylidene-2-deoxy-3-O-p-methoxybenzyl- $\alpha$-D-glucopyranosyl-} $(1 \rightarrow 4)$-methyl $\quad$ 1,2-O- $(S)$-ethylidene- $\beta$-L-idopyranosyluronate $(12)$ - To a stirring solution of donor 11 (35 mg, $67 \mu \mathrm{mol})$, diol acceptor 10 (32 mg, $137 \mu \mathrm{mol})$, NIS (20 mg, $87 \mu \mathrm{mol})$ and $\mathrm{MS}$ in dry dichloromethane $(1.5 \mathrm{~mL})$ at $-20{ }^{\circ} \mathrm{C}$ under argon, trifluromethanesulfonic acid $(0.6 \mu \mathrm{L}, 7 \mu \mathrm{mol})$ was added. The mixture was allowed to warm to $10{ }^{\circ} \mathrm{C}$. The reaction was quenched with saturated $\mathrm{NaHCO}_{3}$ and diluted with dichloromethane. After filtration, the mixture was washed with saturated $\mathrm{NaHCO}_{3}$, $\mathrm{Na}_{2} \mathrm{~S}_{2} \mathrm{O}_{3}(10 \%), \mathrm{H}_{2} \mathrm{O}$, brine, and died over $\mathrm{Na}_{2} \mathrm{SO}_{4}$. The organic layer was then concentrated and the resulting residue was purified by column chromatography using toluene - EtOAc (3:2 v/v) as eluent to give compound 12 (24 mg, 57\%). ${ }^{1} \mathrm{H}$ NMR (400 $\left.\mathrm{MHz}, \mathrm{CDCl}_{3}\right) \delta 7.48-6.82(\mathrm{~m}, 9 \mathrm{H}, \mathrm{PMB}+\mathrm{Ph}), 5.50(\mathrm{~s}, 1 \mathrm{H}, \mathrm{Ph}), 5.33(\mathrm{~d}, J 2.7,1 \mathrm{H}, \mathrm{H}-$ 1), 5.25 (q, $\left.1 \mathrm{H}, J 4.3, \mathrm{CHCH}_{3}\right), 4.92$ (d, 1H, $\left.J 3.3, \mathrm{H}-1^{\prime}\right), 4.79$ (d, 1H, $J$ 10.5, PMB), 4.62 (d, 1H, $J$ 10.5, PMB), 4.53 (bs, 1H, H-5), 4.29 (dd, 1H, $J$ 4.9, 10.0, H-6'a), 3.97 (m, 3H, H-4 + H-3' + H-6'b), 3.75 (m, 7H, H-2 + $\left.\mathrm{CO}_{2} \mathrm{CH}_{3}+\mathrm{PMB}\right), 3.61$ (m, 2H, H-4' + H-5'), $3.35\left(\mathrm{dd}, J 3.5,9.8, \mathrm{H}-2^{2}\right), 2.38(\mathrm{~d}, 1 \mathrm{H}, J 9.23-\mathrm{OH}), 1.68\left(\mathrm{~d}, 3 \mathrm{H}, J 4.6, \mathrm{CHCH}_{3}\right) . \mathrm{m} / \mathrm{z}$ (High Res. MALDI-FTMS) calcd $\mathrm{C}_{30} \mathrm{H}_{35} \mathrm{~N}_{3} \mathrm{O}_{12} \mathrm{Na}^{+}:$: 652.2113, found: 652.2131 . 
2-azido-6-O-acetyl-3-O-benzyl-2-deoxy-4-O-levulinoyl- $\alpha$-D-glucopyranosyl-(1 $\rightarrow 4)$ methyl 1,2-O-(S)-ethylidene- $\beta$-L-idopyranosyluronate (14) - To a stirring solution of donor 13 (116 mg, $214 \mu \mathrm{mol})$, diol acceptor 10 (100 mg, $427 \mu \mathrm{mol})$, NIS (62 mg, $276 \mu \mathrm{mol})$ and $\mathrm{MS}$ in dry dichloromethane $(5 \mathrm{~mL})$ at $-15{ }^{\circ} \mathrm{C}$ under argon, trifluromethanesulfonic acid (cat.) was added. The mixture was allowed to warm to 10 ${ }^{\circ} \mathrm{C}$. The reaction was quenched with saturated $\mathrm{NaHCO}_{3}$ and diluted with dichloromethane. After filtration, the mixture was washed with saturated $\mathrm{NaHCO}_{3}$, $\mathrm{Na}_{2} \mathrm{~S}_{2} \mathrm{O}_{3}(10 \%), \mathrm{H}_{2} \mathrm{O}$, brine, and died over $\mathrm{Na}_{2} \mathrm{SO}_{4}$. The organic layer was then concentrated and the resulting residue was purified by column chromatography using toluene - EtOAc $(3: 2 \mathrm{v} / \mathrm{v})$ as eluent to give compound $14(88 \mathrm{mg}, 63 \%) .{ }^{1} \mathrm{H}$ NMR (400 $\left.\mathrm{MHz}, \mathrm{CDCl}_{3}\right) \delta 7.32(\mathrm{~m}, 5 \mathrm{H}, \mathrm{Ph}), 5.38(\mathrm{~d}, J 2.8,1 \mathrm{H}, \mathrm{H}-1), 5.30\left(\mathrm{q}, 1 \mathrm{H}, J 5.1, \mathrm{CHCH}_{3}\right)$, $5.11\left(\mathrm{dd}, 1 \mathrm{H}, J 9.2,10.3, \mathrm{H}-4^{\prime}\right), 5.03$ (d, 1H, J 3.4, H-1'), 4.76 (d, 1H, J 11.0, Bn), 4.64 (d, $1 \mathrm{H}, J 11.0, \mathrm{Bn}), 4.60(\mathrm{dd}, 1 \mathrm{H}, J 4.0,6.5, \mathrm{H}-3), 4.50(\mathrm{~d}, 1 \mathrm{H}, J 2.0, \mathrm{H}-5), 4.16$ (dd, 1H, $J$ 3.2, 7.4, H-6'a), 4.13 ('t', 1H, J 7.1, H-6'b), 4.04 (m, 2H, H-4 + H-5'), 3.95 (dd, 1H, J 9.4, 10.1, H-3'), 3.82 (dd, 1H, J 2.8, H-2), 3.79 (s, 3H, OMe), 3.36 (dd, J 3.4, 10.3, H-2'), 2.68 (t, 2H, J 6.4, Lev), 2.44 (m, 3H, 3-OH + Lev), 2.16 (s, 3H, Lev), 2.06 (s, 3H, Lev), $1.56\left(\mathrm{~d}, 3 \mathrm{H}, J\right.$ 5.0, $\mathrm{CHCH}_{3}$ ). m/z (High Res. MALDI-FTMS) calcd $\mathrm{C}_{29} \mathrm{H}_{37} \mathrm{~N}_{3} \mathrm{O}_{14} \mathrm{Na}^{+}$: 674.2168, found:674.2150.

2-azido-4,6-O-benzylidene-2-deoxy-3-O-p-methoxybenzyl- $\alpha$-D-glucopyranosyl$(1 \rightarrow 4)$-methyl 1,2-O-(S)-ethylidene- $\alpha$-D-glucopuranosyluronate (26a) and 2-azido4,6-O-benzylidene-2-deoxy-3-O-p-methoxybenzyl- $\alpha$-D-glucopyranosyl-( $1 \rightarrow 3)$-methyl 1,2-O-(S)-ethylidene- $\alpha$-D-glucopuranosyluronate (27a) - To a stirring solution of 
donor 11 (49 mg, $94 \mu \mathrm{mol}$ ), diol acceptor 25a (44 mg, $188 \mu \mathrm{mol})$, NIS (25 mg, $111 \mu \mathrm{mol})$ and $\mathrm{MS}$ in dry dichloromethane $(2 \mathrm{~mL})$ at $-20{ }^{\circ} \mathrm{C}$ under argon, trifluromethanesulfonic acid $(1 \mu \mathrm{L}, 11 \mu \mathrm{mol})$ was added. The mixture was allowed to warm to $10^{\circ} \mathrm{C}$. The reaction was quenched with saturated $\mathrm{NaHCO}_{3}$ and diluted with dichloromethane. After filtration, the mixture was washed with $\mathrm{NaHCO}_{3}, \mathrm{Na}_{2} \mathrm{~S}_{2} \mathrm{O}_{3}, \mathrm{H}_{2} \mathrm{O}$, brine, and died over $\mathrm{Na}_{2} \mathrm{SO} 4$. The organic layer was then concentrated and the resulting residue was purified by column chromatography using toluene - EtOAc $(5: 1 \mathrm{v} / \mathrm{v})$ as eluent to give compound 27a (18 mg, 30\%) and 26a (26 mg, 44\%).

Data for 26a ${ }^{1} \mathrm{H}$ NMR (400 MHz, $\left.\mathrm{CDCl}_{3}\right) \delta 7.50-6.83(\mathrm{~m}, 9 \mathrm{H}, \mathrm{PMB}+\mathrm{Ph}), 5.69(\mathrm{~d}, J$ 4.5, 1H, H-1), 5.58 (s, 1H, CHPh), 5.49 (q, 1H, J 4.7, $\left.\mathrm{CHCH}_{3}\right), 5.12$ (d, 1H, J 3.8, H-1'), 4.89 (d, 1H, J 10.5, PMB), 4.73 (d, 1H, $J$ 10.5, PMB), 4.31 (d, 1H, $J 8.2 \mathrm{~Hz}, \mathrm{H}-5), 4.25$ (dd, 1H, J 4.5, 10.0, H-6'a), 4.15 (dd, 1H, J 4.4, 6.9, H-2), 4.05 (ddd, 1H, J 2.6, 7.0, 9.7, H-3), 4.02 ('t', 1H, J 9.7, H-3'), 3.86 (m, 1H, H-5'), 3.80 (d, 6H, $\left.\mathrm{CO}_{2} \mathrm{CH}_{3}+\mathrm{PMB}\right), 3.70$ (m, 4H, H-6b' + H-4'), 3.61 (dd, J 4.0, 10.0, H-2'), 2.04 (bs, 1H, 3-OH), 1.42 (d, 3H, J 5.0, $\mathrm{CHCH}_{3}$ ). $\mathrm{m} / \mathrm{z}$ (High Res. MALDI-FTMS) calcd $\mathrm{C}_{30} \mathrm{H}_{35} \mathrm{~N}_{3} \mathrm{O}_{12} \mathrm{SNa}^{+}:$652.2113, found: 652.2114 .

Data for 27a ${ }^{1} \mathrm{H}$ NMR (400 MHz, $\left.\mathrm{CDCl}_{3}\right) \delta 7.51-6.85(\mathrm{~m}, 9 \mathrm{H}, \mathrm{PMB}+\mathrm{Ph}), 5.70(\mathrm{~d}, J$ 4.3, 1H, H-1), 5.59 (s, 1H, CHPh), 5.50 (q, 1H, J 4.0, $\left.\mathrm{CHCH}_{3}\right), 5.15$ (d, 1H, J 3.7, H-1'), 4.89 (d, 1H, J 10.5, PMB), 4.74 (d, 1H, J 10.5, PMB), 4.28 (d, 1H, J 8.1Hz, H-5), 4.25 (dd, 1H, J 5.1, 10.3, H-6'a), 4.15 (dd, 1H, J 4.4, 6.3, H-2), 4.05 ('t', 1H, J 9.4, H-3'), 4.04 (overlapped, 1H, H-5'), 3.96 (d't', 1H, J 3.1, 8.2, H-4), 3.84 (dd, 1H, J 2.5, 6.3, H3), 3.82 (s, 3H, $\mathrm{CO}_{2} \mathrm{CH}_{3}$ ), 3.80 (s, 3H, PMB), 3.76 (dd, 1H, J 3.1, 10.3, H-6'b), 3.71 ('t', $\left.1 \mathrm{H}, J 9.5, \mathrm{H}-4^{\prime}\right), 3.54$ (dd, $\left.J 3.8,10.0, \mathrm{H}-2^{\prime}\right), 3.50$ (d, 1H, J 3.5, 4-OH), 1.39 (d, 3H, J 4.5, 
$\mathrm{CHCH}_{3}$ ). m/z (High Res. MALDI-FTMS) calcd $\mathrm{C}_{30} \mathrm{H}_{35} \mathrm{~N}_{3} \mathrm{O}_{12} \mathrm{SNa}^{+}$: 652.2113, found: 652.2121

$p$-methylphenyl $2,3,4,6$-tetra- $O$-acetyl-1-thio- $\beta$-D-mannopyranoside (29) - To an icecold solution of $p$-toluenethiol (26.6 g, $214 \mathrm{mmol})$ in anhydrous DMF (130 mL), was added tert-butoxide (14.4 g, $128 \mathrm{mmol})$, and the mixture was stirred for $15 \mathrm{~min}$. A solution of bromide 28 (44 g, $107 \mathrm{mmol})$ in anhydrous THF $(50 \mathrm{~mL})$ was added dropwise. The mixture was stirred at room temperature for $3 \mathrm{~h}$. The reaction mixture was concentrated and the residue was diluted with EtOAc, washed with $\mathrm{H}_{2} \mathrm{O}$, brine, dried over anhydrous $\mathrm{Na}_{2} \mathrm{SO}_{4}$ and concentrated. Crystallization with EtOAc-hexane gave 29 (46 g, 94\%). ${ }^{1} \mathrm{H}$ NMR (300 MHz, $\left.\mathrm{CD}_{3} \mathrm{OD}\right) \delta 7.42-7.09$ (m, 4H, Tol), 5.64 (dd, $1 \mathrm{H}, J$ 1.2, 3.6, H-2), 5.26 ('t', 1H, $J$ 9.9, H-4), 5.03 (dd, 1H, $J 3.6,10.2, \mathrm{H}-3$ ), 4.83 (d, 1H, $J$ 0.9, H-1), 4.25 (dd, 1H, $J$ 6.3, 12, H-6a), 4.15 (dd, 1H, $J$ 2.7, 12.3, H-6b), 3.66 (ddd, 1H, $J$ 2.7, 6.6, 10.2, H-5), 2.34 (s, 3H, Tol), 2.21 (s, 3H, OAc), 2.09 (s, 3H, OAc), 2.03 (s, 3H, OAc), 1.98 (s, 3H, OAc). m/z (High Res. MALDI-FTMS) calcd $\mathrm{C}_{21} \mathrm{H}_{26} \mathrm{O}_{9} \mathrm{SNa}^{+}:$477.1190, found: 477.1190 .

p-methylphenyl 1-thio- $\beta$-D-mannopyranoside (30) - Compound 29 (20 g, 44 mmol) was suspended in dry $\mathrm{MeOH}(250 \mathrm{~mL})$ and $\mathrm{NaOMe}$ (cat.) was added. The mixture was stirred overnight and neutralized to $\mathrm{pH} 1$ with $\mathrm{H}^{+}$resin. After filtration, the mixture was concentrated and crystallization with $\mathrm{MeOH}-\mathrm{H}_{2} \mathrm{O}$ at $4{ }^{\circ} \mathrm{C}$ afford $30(11.5 \mathrm{~g}, 92 \%){ }^{1} \mathrm{H}$ NMR (400 MHz, $\left.\mathrm{CD}_{3} \mathrm{OD}\right) \delta 4.88(\mathrm{~d}, 1 \mathrm{H}, J 1.2, \mathrm{H}-1), 4.00(\mathrm{dd}, 1 \mathrm{H}, J 0.8,3.6, \mathrm{H}-2), 3.83$ (dd, 1H, $J$ 2.8, 12.4, H-6a), 3.69 (dd, 1H, J 5.6, 12.0, H-6b), 3.59 ('t', 1H, $J$ 9.6, H-4), 
3.45 (dd, 1H, $J$ 3.6, 9.6, H-3), 3.22 (ddd, $1 \mathrm{H}, J$ 2.8, 6.0, 9.6, H-5), 2.34 (s, 3H, Tol). m/z (High Res. MALDI-FTMS) calcd $\mathrm{C}_{13} \mathrm{H}_{18} \mathrm{O}_{5} \mathrm{SNa}^{+}: 309.0767$, found: 309.0769 .

p-methylphenyl 3-O-p-methoxybenzyl-1-thio- $\beta$-D-mannopyranoside (31) - To a solution of $\mathbf{3 0}(2.3 \mathrm{~g}, 8.0 \mathrm{mmol})$ in anhydrous toluene $(100 \mathrm{~mL})$, was added di- $n$-butyltin oxide ( $2.2 \mathrm{~g}, 8.8 \mathrm{mmol})$; was refluxed with azeotropic removal of water for $6 \mathrm{~h}$ using Dean-Stark tube. The reaction was cooled to $60^{\circ} \mathrm{C}$, tetra- $n$-butylammonium bromide (2.9 $\mathrm{g}, 8.9 \mathrm{mmol})$ and 4-methoxybenzyl chloride $(1.7 \mathrm{~mL}, 12.5 \mathrm{mmol})$ were added the reaction was stirred for overnight at the same temperature. The mixture was concentrated and the residue was purified by column chromatography on silica gel (MeOH-EtOAc, $49: 1 \mathrm{v} / \mathrm{v})$, to give compound 31 (2.1 g, 65\%). ${ }^{1} \mathrm{H}$ NMR (400 MHz, $\left.\mathrm{CDCl}_{3}\right) \delta 7.40-6.88$ (m, 4H, PMB), 4.77 (d, 1H, $J$ 1.2, H-1), 4.68 (d, 1H, $J$ 11.2, PMB), 4.53 (d, 1H, $J$ 11.6, PMB), 4.25 (dd, 1H, $J$ 0.8, 3.2, H-2), 3.97 ('t', $1 \mathrm{H}, J$ 9.6, H-4), 3.86 (dd, 1H, $J$ 3.6, 8.8, H-6a), 3.80 (s, 3H, OMe), 3.77 (dd, 1H, J 4.8, 8.5, H-6b), 3.41 (dd, 1H, J 3.2, 9.2, H-3), 3.22 (ddd, 1H, J 3.6, 4.8, 10.0, H-5), 2.33 (s, 3H, Tol). ). m/z (High Res. MALDI-FTMS) calcd $\mathrm{C}_{21} \mathrm{H}_{26} \mathrm{O}_{6} \mathrm{SNa}^{+}: 429.1342$, found: 429.1348 .

p-methylphenyl 4,6-O-benzylidene-3-O-p-methoxybenzyl-1-thio- $\beta$-Dmannopyranoside (32) - To a solution of compound 31 (1.9 g, $4.7 \mathrm{mmol})$ and benzaldehyde dimethyl acetal $(2.1 \mathrm{~mL}, 14.0 \mathrm{mmol})$ in dry $\mathrm{CH}_{3} \mathrm{CN}$, was add camphor sulfonic acid (100mg, $0.43 \mathrm{mmol})$. The reaction was continued for $3.5 \mathrm{~h}$ and then quenched with triethyl amine. Concentration of the mixture followed by column chromatography on silica gel (toluene-EtOAc, $5: 1 \mathrm{v} / \mathrm{v})$, to give compound $32(2.1 \mathrm{~g}$, 
89\%). ${ }^{1} \mathrm{H}$ NMR (400 MHz, $\left.\mathrm{CDCl}_{3}\right) \delta 7.50-6.85(\mathrm{~m}, 9 \mathrm{H}, \mathrm{PMB}+\mathrm{Ph}), 5.60(\mathrm{~s}, 1 \mathrm{H}, \mathrm{Ph})$, 4.80 (s, 1H, H-1), 4.77 (d, 1H, $J$ 11.6, PMB), 4.67 (d, 1H, $J$ 11.6, PMB), 4.31 (dd, 1H, $J$ 5.2, 10.4, H-6a), 4.26 (d, 1H, J 3.2, H-2), 4.14 ('t', 1H, J 9.6, H-4), 3.91 ('t', 1H, J 10.4, H-6b), 3.80 (s, 3H, OMe), 3.66 (dd, 1H, J 3.2, 9.2, H-3), 3.22 (d't', 1H, J 4.8, 10.0, H-5), 2.33 (s, 3H, Tol). m/z (High Res. MALDI-FTMS) calcd $\mathrm{C}_{28} \mathrm{H}_{30} \mathrm{O}_{6} \mathrm{SNa}^{+}: 517.1655$, found: 517.1657. 